\title{
Un nouveau suppresseur de tumeur impliqué dans le modelage de la structure chromatinienne
}

Les tumeurs rhabdoïdes malignes sont des cancers rares, fréquemment métastatiques, résistants aux thérapeutiques actuelles et d'évolution souvent fatale. Initialement décrites au niveau du rein comme des variants particulièrement agressifs de néphroblastome du nourrisson, elles ont par la suite été décrites, parfois chez l'enfant plus âgé voire chez l'adulte, dans des localisations variées, en particulier au niveau du système nerveux central et des tissus mous. L'origine embryonnaire de ces tumeurs reste inconnue.

Au niveau microscopique, les cellules rhabdoïdes sont définies par plusieurs critères dont aucun n'est réellement spécifique d'où de fréquentes difficultés diagnostiques: les cellules sont rondes ou polygonales avec un noyau vésiculaire et un nucléole proéminent, un cytoplasme abondant contenant fréquemment des inclusions acidophiles correspondant, en microscopie électronique, à des amas de filaments intermédiaires. Ces cellules peuvent exprimer des marqueurs de différenciation variés et disparates, de types neuroectodermiques, mésenchymateux ou épithéliaux. Néanmoins, une double positivité pour la vimentine et la kératine ainsi qu'une négativité pour la desmine sont fréquemment notées [1]. $\mathrm{Au}$ niveau génétique, des altérations du chromosome 22, à type, soit de translocations apparemment équilibrées avec des partenaires variés, soit de monosomie complète ou partielle, sont retrouvées dans $60 \%$ à $80 \%$ des cas. L'étude de la partie proximale du chromosome 22 à l'aide de microsatellites et/ou en utilisant les techniques d'hybridation in situ fluorescente a montré non seulement l'existence de pertes d'hétérozygotie, mais également de délétions homozy- suggérant ainsi que l'oncogenèse des tumeurs rhabdoïdes fait intervenir l'inactivation d'un gène suppresseur de tumeur à ce niveau (pour une revue voir [2]).

Nous avons procédé à l'étude systématique de cette région chromosomique au niveau de l'ADN de treize lignées cellulaires de tumeurs rhabdoïdes malignes [3]. Dans cinq cas, on a observé des délétions homozygotes permettant de délimiter une région commune de délétion d'environ $150 \mathrm{~kb}$ codant pour plusieurs gènes dont le gène $h S N F 5 / I N I 1$, préalablement identifié par deux groupes indépendants $[4,5]$. Une sixième lignée s'est avérée particulièrement informative dans cette approche de clonage positionnel. En effet, elle présente un réarrangement visible en Southern blot du gène hSNF5/INI1. Une caractérisation plus précise de ce réarrangement a montré qu'il résultait d'une délétion homozygote limitée aux exons 4 et 5 de ce gène. L'analyse de la séquence du gène hSNF5/INI1 dans les sept autres lignées ne présentant pas de délétion homozygote a permis d'identifier six mutations ponctuelles. Ces mutations, de type non-sens dans un cas, d'insertions ou de délétions de quelques nucléotides dans les autres cas, interrompent le cadre de lecture et sont toujours associées à la perte complète de l'autre allèle du gène $h S N F 5 / I N I 1$. On les observe non seulement au niveau de l'ADN de lignées cellulaires, mais également au niveau des tumeurs fraîches correspondant à ces lignées. En revanche, on ne les retrouve pas au niveau de l'ADN constitutionnel de ces patients, ce qui démontre ainsi leur lien avec l'apparition du phénotype tumoral. Une lésion des deux allèles du gène $h S N F 5 / I N I 1$ a donc pu être observée dans douze tumeurs rhabdoïdes malignes sur treize, suggérant fortement que la perte de fonction de hSNF5/INI1 constitue une étape cruciale de l'oncogenèse de ces tumeurs [3]. Ces différentes altérations sont schématisées sur la figure 1. Il est probable que les études futures permettront de détecter des mutations constitutionnelles du gène hSNF5/ INI1 responsables des cas plurifocaux et/ou familiaux qui ont été observés. La protéine hSNF5/INI1 est une composante des complexes SWI/SNF [6]. Les gènes $S W I$ et $S N F$ ont été identifiés initialement chez la levure car ils étaient nécessaires, d'une part à l'induction du gène $H O$, qui code pour une endonucléase impliquée dans la détermination du type sexuel (d'où le nom SWI, pour mating-type switch, donné à ces gènes) et, d'autre part, à l'expression de l'invertase SUC2, enzyme intervenant dans le catabolisme du sucrose (d'où le nom de $S N F$ pour sucrose non-fermenting de ces gènes). Le lien entre ces protéines et la structure de la chromatine a été initialement établi lorsqu'on a mis en évidence puis caractérisé des mutants suppresseurs de SWI ou SNF. Ces mutations suppressives affectent des histones ou d'autres protéines chromatiniennes. Les protéines SWI/SNF sont très conservées entre espèces de la levure à l'homme et sont associées en complexes de huit à douze sous-unités suivant l'organisme et le type cellulaire considéré. Ces complexes, en facilitant l'accessibilité de facteurs de transcription à leurs cibles spécifiques au niveau de l'ADN, semblent être des co-facteurs essentiels à l'activation transcriptionnelle d'une gamme variée de gènes inductibles $\left(\mathrm{m} / \mathrm{s}\right.$ 1993, $n^{\circ} 1$, p. 106). Leur mode d'action repose sur une modification de la structure des nucléosomes nécessitant l'hydrolyse d'ATP [7]. 


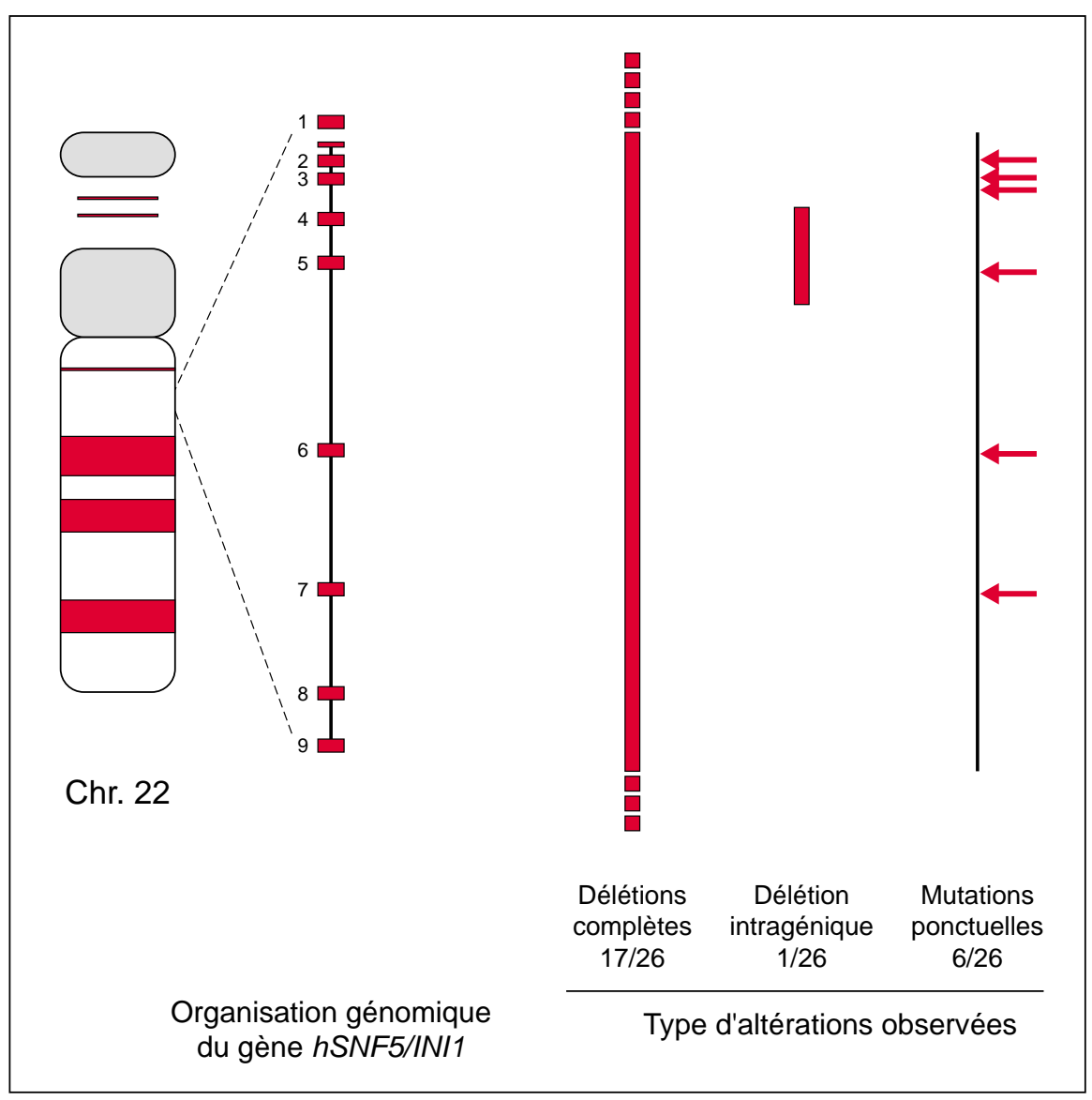

Figure 1. Représentation schématique du chromosome 22, du gène hSNF5/INI1 et des différentes mutations observées dans treize cas de tumeurs rhabdoïdes, soit 26 allèles du gène sur lesquels 24 altérations sont observées.

Dans les tumeurs rhabdoïdes malignes, l'absence de la protéine hSNF5/INI1 pourrait perturber la fonction du ou des complexe(s) SWI/SNF, compromettant ainsi l'expression de gènes impliqués dans la régulation de la prolifération cellulaire, de la différenciation ou de l'apoptose. Le rôle d'un possible déficit de la fonction du complexe SWI/SNF en oncogenèse humaine est-il limité aux tumeurs rhabdoïdes? La délétion du chromosome 22 constitue une altération fréquemment observée dans les cancers. Les analyses du locus $h S N F 5 / I N I 1$ dans ces tumeurs devraient permettre de préciser rapidement le spectre des tumeurs dans lesquelles des mutations $h$ SNF5/INI1 sont observées. D'autres membres des complexes SWI/SNF pourraient aussi être la cible de mutations dans certains cancers. Cette hypothèse devra L'étude du rôle d'anomalies de la régulation de la structure chromatinienne dans le développement de tumeurs constitue une nouvelle voie de recherche en cancérologie. Outre les protéines mentionnées ci-dessus, les modifications post-traductionnelles des histones constituent un autre mode de modulation de la structure chromatinienne qui a été récemment identifié comme partie intégrante du mécanisme d'action de certains suppresseurs de tumeur ou d'oncoprotéines [9-13].

O.D.

I.V.

N.S.

J.L.

A.A.

1. Wick MR, Ritter JH, Dehner LP. Malignant rhabdoid tumours: a clinicopathologic review and conceptual discussion. Semin Diagn Pathol $1995 ; 12$ : 233-48.

2. Rosty C, Peter M, Zucman J, Validire P, Delattre $\mathrm{O}$, Aurias A. Cytogenetic and molecular analysis of a $t(1 ; 22)(p 36 ; 11.2)$ in a rhabdoid tumor with a homozygous deletion of chromosome 22. Genes Chromosome Cancer 1998; 21 : 82-9.

3. Versteege I, Sevenet N, Lange J, RousseauMerck MF, Ambros P, Handgretinger R, Aurias A, Delattre O. Truncating mutations of hNF5/INI1 in aggressive paediatric cancers. Nature 1998; 394: 203-6.

4. Muchardt C, Sardet C, Bourachot B, Onufryk C, Yaniv M. A human protein with homology to Saccharomyces cerevisice SNF5 interacts with the potential helicase hbrm. Nucleic Acids Res 1995; $23: 1127-32$.

5. Kalpana GV, Marmon S, Wang W, Crabtree GR, Goff SP. Binding and stimulation of HIV-1 integrase by a human homolog of yeast transcription factor SNF5. Science 1994; 266 : 2002-6.

6. Peterson CL. Multiple switches to turn on chromatin? Curr Opin Genet Dev 1996; 6: 171-5.

7. Schnitzler G, Sif S, Kingston RE. Human SWI/SNF interconverts a nucleosome between its base state and a stable remodeled state. Cell 1998; 94: 17-27.

8. Rozenblatt-Rosen O, Rozovskaia T, Burakov D, Sedkov Y, Tillib S, Blechman J, Nakamura T, Croce CM, Mazo A, Canaani E. The C-terminal SET domains of ALL-1 and TRITHORAX interact with the INI1 and SNR1 proteins, components of the SWI/SNF complex. Proc Natl Acad Sci USA 1998; 95: 4152-7.

9. Magnaghi-Jaulin L, Groisman R, Naguibneva I, Robin P, Lorain S, Le Villain JP, Troalen F, Trouche D, Harel-Bellan A. Retinoblastoma protein represses transcription by recruiting a histone deacetylase. Nature 1998; 391: 601-4.

10. Magnaghi-Jaulin L, Groisman R, Naguibneva I, Robin P, Trouche D, Harel-Bellan A. Rôle des histone-désacétylases dans le contrôle de la prolifération cellulaire. Med Sci 1998; 14: 455-7.

11. Brehm A, Miska EA, McCance DJ, Reid JL, Bannister AJ, Kouzarides T. Retinoblastoma protein recruits histone deacetylase to repress transcription. Nature 1998; 391 : 597-601.

12. Lin RJ, Nagy L, Inoue S, Shao W, Miller WH Jr, Evans RM. Role of histone deacetylase complex in acute promyelocytic leukaemia. Nature 1998; 391: 811-4.

13. Grignani F, De Matteis S, Nervi C, et al. Fusion proteins of the retinoic acid receptor- $\alpha$ recruit histone deacetylase in promyelocytic leukaemia. Nature 1998; 391 : 815-8.
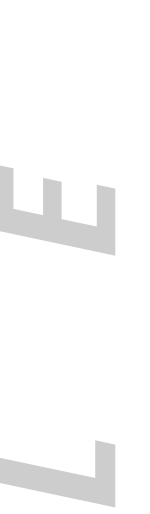

(1)

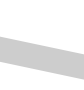

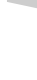

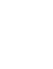

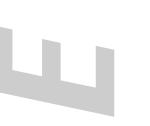

\author{
.
}

(

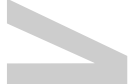

.

(1)

r

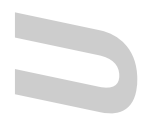

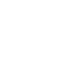

